\title{
A regularization method for solving the Poisson equation for mixed unbounded- periodic domains
}

Spietz, Henrik Juul ; Mølholm Hejlesen, Mads; Walther, Jens Honoré

Published in:

Journal of Computational Physics

Link to article, DOI:

10.1016/j.jcp.2017.12.018

Publication date:

2018

Document Version

Peer reviewed version

Link back to DTU Orbit

Citation (APA):

Spietz, H. J., Mølholm Hejlesen, M., \& Walther, J. H. (2018). A regularization method for solving the Poisson equation for mixed unbounded-periodic domains. Journal of Computational Physics, 356, 439-447.

https://doi.org/10.1016/j.jcp.2017.12.018

\section{General rights}

Copyright and moral rights for the publications made accessible in the public portal are retained by the authors and/or other copyright owners and it is a condition of accessing publications that users recognise and abide by the legal requirements associated with these rights.

- Users may download and print one copy of any publication from the public portal for the purpose of private study or research.

- You may not further distribute the material or use it for any profit-making activity or commercial gain

- You may freely distribute the URL identifying the publication in the public portal 


\section{Accepted Manuscript}

A regularization method for solving the Poisson equation for mixed unbounded-periodic domains

Henrik Juul Spietz, Mads Mølholm Hejlesen, Jens Honoré Walther

$\begin{array}{ll}\text { PII: } & \text { S0021-9991(17)30903-8 } \\ \text { DOI: } & \text { https://doi.org/10.1016/j.jcp.2017.12.018 } \\ \text { Reference: } & \text { YJCPH 7756 }\end{array}$

Reference: $\quad$ YJCPH 7756

To appear in: Journal of Computational Physics

Received date: 20 July 2017

Revised date: 7 December 2017

Accepted date: 11 December 2017

Please cite this article in press as: H. Juul Spietz et al., A regularization method for solving the Poisson equation for mixed unbounded-periodic domains, J. Comput. Phys. (2017), https://doi.org/10.1016/j.jcp.2017.12.018

This is a PDF file of an unedited manuscript that has been accepted for publication. As a service to our customers we are providing this early version of the manuscript. The manuscript will undergo copyediting, typesetting, and review of the resulting proof before it is published in its final form. Please note that during the production process errors may be discovered which could affect the content, and all legal disclaimers that apply to the journal pertain. 


\title{
A regularization method for solving the Poisson equation for mixed unbounded-periodic domains
}

\author{
Henrik Juul Spietz ${ }^{\mathrm{a}}$, Mads Mølholm Hejlesen ${ }^{\mathrm{a}}$, Jens Honoré Walther ${ }^{\mathrm{a}, \mathrm{b}, *}$ \\ ${ }^{a}$ Department of Mechanical Engineering, Technical University of Denmark, Building 403, DK-2800 Kgs. Lyngby, Denmark \\ ${ }^{b}$ Computational Science and Engineering Laboratory, ETH Zürich, Clausiusstrasse 33, CH-8092 Zürich, Switzerland
}

\begin{abstract}
Regularized Green's functions for mixed unbounded-periodic domains are derived. The regularization of the Green's function removes its singularity by introducing a regularization radius which is related to the discretization length and hence imposes a minimum resolved scale. In this way the regularized unboundedperiodic Green's functions can be implemented in an FFT-based Poisson solver to obtain a convergence rate corresponding to the regularization order of the Green's function. The high order is achieved without any additional computational cost from the conventional FFT-based Poisson solver and enables the calculation of the derivative of the solution to the same high order by direct spectral differentiation. We illustrate an application of the FFT-based Poisson solver by using it with a vortex particle mesh method for the approximation of incompressible flow for a problem with a single periodic and two unbounded directions.

Keywords: The Poisson equation, unbounded and periodic domains, mixed boundary conditions, regularization methods, Green's function solution, Vortex methods
\end{abstract}

\section{Introduction}

The Poisson equation is an elliptic equation that most commonly appear in physics when solving potential fields. Examples are the potential of gravitational or electrical charge interactions, or the velocity field induced by a vortex in a potential flow. In this work we propose a high order solver for the Poisson equation for mixed unbounded-periodic domains, which are domains having a mixture of unbounded and periodic directions based on a Green's function approach. In terms of boundary conditions we may use the phrase mixed periodic and free-space boundary conditions.

Using Green's functions is the preferred method for solving linear differential equations in an unbounded domain. The Green's function represents a homogeneous solution which is derived analytically with the appropriate boundary conditions, and then used to obtain the particular solution by a convolution with the right-hand-side field of the Poisson equation.

${ }^{*}$ Corresponding author at: Department of Mechanical Engineering, Technical University of Denmark, Building 403, DK-2800 Kgs. Lyngby, Denmark. Tel.: + 454525 4327; fax: + 454588 4325. E-mail address: jhw@mek.dtu.dk (J. H. Walther). 
For triple periodic systems it is widely used within the field of electrostatics to decompose the electrostatic potential into a singular short-range term and a smooth long-range term. This technique is known as Ewald summation [1]. It constitutes an $N$-body problem, where $N$ is the number of charged particles contained within the unit domain. The summation may be calculated using particle-particle particle-mesh like methods [2], where the long-range term is computed in Fourier space using an FFT-based particle-mesh method with computational efforts scaling $\mathcal{O}(N \log N)$ (see e.g., [3, 4]). Likewise, expressions for the Ewald sums for the cases of mixed periodic and free-space boundary conditions have been derived in [5] also applicable for fast summation methods $[6,7]$. The Ewald summation technique, however does not provide an explicit Green's function, which may be discretized and used with a generic solution procedure such as the FFT-based solver presented in the following.

A high-order FFT solver for the unbounded Poisson equation has recently been presented in references $[8,9,10]$. Here the high order is achieved by deriving regularized Green's functions by removing its singularity through the introduction of a minimum resolved length scale. The method is based on the work of references $[11,12,13,14,15]$ who employ regularized particles for mesh free particle interactions. We note that solvers based on the adaptive Fast Multipole Method (FMM) [16, 17] are alternatives to the FFT-based solvers. The FMM based solvers have an advantage in systems with highly non-uniform particle distributions, since they are not restricted to uniform meshes (see [18] for a detailed comparison of computational efforts required for the two methods). For details on higher-order FMM methods for partial differential equations in general the reader is referred to the references $[19,20]$.

A simple approach to obtain a Green's function for unbounded-periodic domains is to use the method of repeating domains where the unbounded Green's function is summed to implicitly account for a specified number of repeated fields and thus obtain a semi-periodic solution [21]. With the FMM this is effectively employed, since only a local multipole expansion of the unit domain need to be summed to account for the well-separated repeated domains with insignificant additional computational efforts [16]. For a mesh-based FFT-solver however, the method impose a growth of the efforts associated with the precomputation of the convolution kernel, that scales linearly with the number of repeated domains. In both cases it introduces an additional parameter in the algorithm which influences the overall accuracy of the method. The high order regularized Green's functions for unbounded domains derived in references [8, 9, 10] may be used together with the method of repeating domains. For this approach the error due to the semi-periodic approximation convergences with respect to the number of repeated domains as first and third order in two- and three dimensions (2D and 3D), respectively [10]. This corresponds to the rate of decay of the free-space Green's functions in the two spatial dimensions. Hence it may be well worth to look for analytic Green's functions which implicitly account for the periodicity.

A more formal approach to achieve a Green's function for unbounded-periodic domains was proposed by Chatelain and Koumoutsakos [22]. Here the Poisson equation was solved directly in the periodic directions 
yielding a modified Helmholtz equation for the unbounded directions which in turn is solved by deriving the appropriate real space Green's functions.

In the present work we adopt the approach of Chatelain and Koumoutsakos [22] by deriving Green's functions which are functions of Fourier space variables in the periodic directions and real space variables in the unbounded directions. Combined with the regularization method presented in $[8,9,10]$ we derive regularized Green's functions for unbounded-periodic domains. The regularized unbounded-periodic Green's functions are implemented in an FFT-based Poisson solver using the zero-padding method of Hockney and Eastwood [2] to obtain a linear convolution in the unbounded directions. We show that the solver obtains a convergence rate corresponding to the regularization order of the Green's function.

For a single free-space and one or more periodic boundary conditions, we note that solving the Poisson equation essentially reduces to solving a one-dimensional modified Helmholtz equation with free-space boundary conditions for each wave number set (when having Fourier transformed the problem in the period directions). A spectrally accurate procedure for doing this has been outlined in [23, 24]. This procedure is not applicable when the system has more than one free-space condition.

\section{Methodology}

The Poisson equation is formally stated as

$$
\nabla^{2} A(\boldsymbol{x})=-B(\boldsymbol{x})
$$

where $B(\boldsymbol{x})$ is a known bounded field and $A(\boldsymbol{x})$ is the desired solution field. As $B(\boldsymbol{x})$ is bounded we may state for the unbounded domains, that

$$
A(x) \rightarrow 0 \text { for }|\boldsymbol{x}| \rightarrow \infty
$$

and for the periodic domains that

$$
A(\boldsymbol{x})=A(\boldsymbol{x}+n \boldsymbol{L})
$$

Here $\boldsymbol{L}$ is the length of the domain and $n \in \mathbb{Z}$. In this work we seek the solution of Eq. (1) for an unbounded-periodic domain which is subject to the conditions of Eqs. (2) and (3) for the unbounded and periodic directions, respectively.

In many applications such as astrophysics, electrodynamics and vortex dynamics, the vector field to be solved $\boldsymbol{v}(\boldsymbol{x})$ is described by potential functions using the Helmholtz decomposition

$$
\boldsymbol{v}(\boldsymbol{x})=\boldsymbol{\nabla} \times \boldsymbol{\psi}(\boldsymbol{x})-\boldsymbol{\nabla} \phi(\boldsymbol{x}) \quad \text { where } \quad \boldsymbol{\nabla} \cdot \boldsymbol{\psi}(\boldsymbol{x})=0
$$

The fundamental operations describing the conservation of the flux and circulation of the vector field $\boldsymbol{v}$ is the divergence $\vartheta(\boldsymbol{x})=\boldsymbol{\nabla} \cdot \boldsymbol{v}(\boldsymbol{x})$ and the curl $\boldsymbol{\omega}(\boldsymbol{x})=\boldsymbol{\nabla} \times \boldsymbol{v}(\boldsymbol{x})$, respectively. From Eq. (4) it follows that 
these may be expressed by the potential functions as

$$
\vartheta(\boldsymbol{x})=-\nabla^{2} \phi(\boldsymbol{x}) \quad \text { and } \quad \boldsymbol{\omega}(\boldsymbol{x})=-\nabla^{2} \boldsymbol{\psi}(\boldsymbol{x}) .
$$

As seen, both potentials may be obtained by solving the Poisson equation. By utilizing the linearity of the Poisson equation and considering the equations by their Fourier transform we obtain the algebraic equations

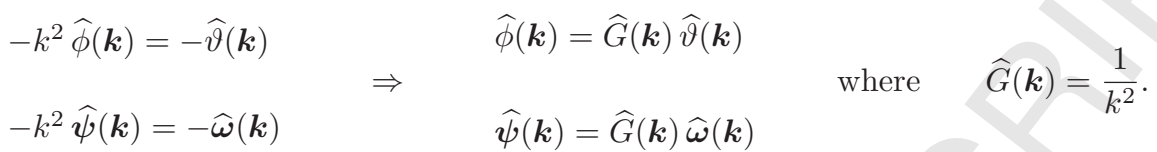

Here $\hat{\imath}$ denotes a Fourier transformed variable, $\iota$ the imaginary unit, $\boldsymbol{k}$ is the angular wave-number of the Fourier space, and $k=|\boldsymbol{k}|$ its modulus. $\widehat{G}(\boldsymbol{k})$ is the Green's function in Fourier space which represents the homogeneous solution to the Poisson equation. We may furthermore obtain the vector field of Eq. (4) directly by incorporating the gradient operator into the Green's function as $\boldsymbol{K}(\boldsymbol{x})=\boldsymbol{\nabla} G(\boldsymbol{x})$ after which

$$
\begin{array}{lll}
-k^{2} \widehat{\boldsymbol{v}}_{\vartheta}(\boldsymbol{k})=-\iota \boldsymbol{k} \widehat{\vartheta}(\boldsymbol{k}) & \widehat{\boldsymbol{v}}_{\vartheta}(\boldsymbol{k})=\widehat{\boldsymbol{K}}(\boldsymbol{k}) \widehat{\vartheta}(\boldsymbol{k}) \\
-k^{2} \widehat{\boldsymbol{v}}_{\omega}(\boldsymbol{k})=-\iota \boldsymbol{k} \times \widehat{\boldsymbol{\omega}}(\boldsymbol{k})
\end{array} \quad \Rightarrow \quad \begin{aligned}
& \widehat{\boldsymbol{v}}_{\omega}(\boldsymbol{k})=\widehat{\boldsymbol{K}}(\boldsymbol{k}) \times \widehat{\boldsymbol{\omega}}(\boldsymbol{k}) \\
& \text { where }
\end{aligned} \quad \widehat{\boldsymbol{K}}(\boldsymbol{k})=\frac{\iota \boldsymbol{k}}{k^{2}} .
$$

It is seen that the solution to the Poisson equations in Eqs. (6) and (7) is obtained by means of a convolution between the Green's function and the right-hand-side of the Poisson equation. The linear convolution needed in the unbounded directions is achieved by the zero-padding method of Hockney and Eastwood [2].

The real space Green's function is obtained by the inverse Fourier transform using the appropriate integration intervals for each direction i.e. an unbounded interval for the unbounded directions and a periodic interval for the periodic directions. However, for a discrete approximation, such as is used in numerical simulations, the discretization of the domain in the physical space, results in a bounded domain in the Fourier space, represented by the set of wave-numbers $k_{i}=-k_{s} / 2, \ldots, k_{s} / 2$. Here $k_{s}=2 \pi / h$ is the angular sampling wave-number corresponding to the discretization length $h$ and $k_{s} / 2$ is the highest resolved angular wave-number due to the Nyquist-Shannon sampling theorem. The exact Green's function of Eq. (6) can thus not be represented in a discretized space and using it will introduce errors in the calculation with a limited convergence rate of $\mathcal{O}\left(h^{2}\right)$ cf. Rasmussen [25].

In order to amend this and derive a method to achieve a higher order convergence rate is to use a spectrally compact regularization function which makes the integral of the inverse Fourier transform convergent within its integration interval. Hence the regularization function should to be chosen to fulfill

$$
\widehat{\zeta}(k)=1 \quad \text { for } \quad k=0 \quad \text { and } \quad \widehat{\zeta}(k)=0 \text { for } \quad k \geq k_{s} / 2
$$

13 This leads to a regularized Green's function which is bounded by a maximum wavenumber and the corre- 
sponding homogeneous equation becomes

$$
\widehat{G}(k)=\frac{\widehat{\zeta}(k)}{k^{2}} \quad \Rightarrow \quad \nabla^{2} G(r)=-\zeta(r) .
$$

The order of the regularization function is increased to $m$-th order by nullifying the $m-2$ order derivatives of the spectral function at $k=0$. This is equivalent to conserving the higher order moments in real space which is summarized by the following additional requirements

$$
\left.\frac{d^{\beta} \widehat{\zeta}}{d k^{\beta}}\right|_{k=0}=\int_{-\infty}^{\infty} r^{\beta} \zeta(r) d \boldsymbol{x}=0^{\beta} \quad \text { for } \quad \beta=0,2, \ldots, m-2 .
$$

We note that $m$ is an even number and that the moments for odd integers of $\beta$ are automatically satisfied due to the radial symmetry of the function.

Following Hejlesen et al. $[8,9,10]$ we obtain a high order regularization function $\zeta_{m}$ of order $m$ by performing an approximate deconvolution which effectively conserves higher order moments of the original field. A high order Gaussian regularization function can thus be obtained from a Gaussian function by

$$
\widehat{\zeta}_{m}(s)=\widehat{D}_{m}(s) \exp \left(-\frac{s^{2}}{2}\right) \quad \text { where } \quad \widehat{D}_{m}(s)=\sum_{n=0}^{m / 2-1} \frac{\left(s^{2} / 2\right)^{n}}{n !} .
$$

Here $s=k \sigma$ is the radial angular wave-number normalized with $\sigma$, which represents a smoothing radius. $\widehat{D}_{m}$ is an $m$-th order approximation of the inverse Gaussian function.

As $k_{s}$ is proportional to $1 / h$, and the extent of the Gaussian regularization function in Fourier space is proportional to $1 / \sigma$ the formal choice of smoothing radius is $\sigma=\alpha h$ where $\alpha=2$. In this way the bounds of the Fourier space (i.e. $k_{s} / 2$ ) and the extent of the Gaussian regularization function scales equally, resulting in a constant truncation error when varying $h$.

We now derive the regularized Green's functions for the Poisson equation in a mixed periodic and unbounded domain in $2 \mathrm{D}$, and then extend the result to the 3D case with two periodic and one unbounded direction. In $2 \mathrm{D}$ with one periodic (the $y$-direction) and one unbounded (the $x$-direction) boundary conditions, the regularized Green's function is found by the inverse Fourier transform

$$
G_{m}(x, y)=\frac{1}{2 \pi} \int_{-k_{s} / 2}^{k_{s} / 2}\left(\frac{1}{2 \pi} \int_{-\infty}^{\infty} \frac{\widehat{\zeta}_{m}\left(\sqrt{k_{x}^{2}+k_{y}^{2}}\right)}{k_{x}^{2}+k_{y}^{2}} \exp \left(\iota k_{x} x\right) d k_{x}\right) \exp \left(\iota k_{y} y\right) d k_{y} .
$$

For the Green's function which has been Fourier transformed in the $y$-direction and using normalized coordinates, we have

$$
\widehat{G}_{m}\left(\rho_{x}, s_{y}\right)=\frac{\sigma}{2 \pi} \int_{-\infty}^{\infty} \frac{\widehat{\zeta}_{m}\left(\sqrt{s_{x}^{2}+s_{y}^{2}}\right)}{s_{x}^{2}+s_{y}^{2}} \exp \left(\iota s_{x} \rho_{x}\right) d s_{x},
$$

where $s_{x}=\sigma k_{x}, s_{y}=\sigma k_{y}$, and $\rho_{x}=|x| / \sigma$. Inserting the Gaussian regularization function on the generic form (Eq. (11)) we obtain

$$
\widehat{G}_{m}\left(\rho_{x}, s_{y}\right)=\frac{\sigma}{2 \pi} \exp \left(-\frac{\rho_{x}^{2}+s_{y}^{2}}{2}\right) \int_{-\infty}^{\infty} \frac{D_{m}\left(\sqrt{s_{x}^{2}+s_{y}^{2}}\right)}{s_{x}^{2}+s_{y}^{2}} \exp \left(-\frac{\left(s_{x}-\iota \rho_{x}\right)^{2}}{2}\right) d s_{x} .
$$


By changing to radial variables and using integration by parts this integral may be found on the general form

$$
\begin{aligned}
\widehat{G}_{m}(\rho, s) & =\frac{\sigma}{4 s}\left[\left(1-\operatorname{erf}\left(\frac{s-\rho}{\sqrt{2}}\right)\right) \exp (-s \rho)+\left(1-\operatorname{erf}\left(\frac{s+\rho}{\sqrt{2}}\right)\right) \exp (s \rho)\right] \\
& +\frac{\sigma \sqrt{2}}{\sqrt{\pi}} P_{m}(s, \rho) \exp \left(-\frac{s^{2}+\rho^{2}}{2}\right) .
\end{aligned}
$$

Here $P_{m}(s, \rho)$ is a polynomial which for $m=\{2,4,6,8,10\}$ is given by

$$
\begin{aligned}
P_{2}(s, \rho) & =0 & P_{8}(s, \rho) & =\frac{11}{32}+\frac{1}{12} s^{2}-\frac{1}{8} \rho^{2}-\frac{1}{48} s^{2} \rho^{2}+\frac{1}{96}\left(s^{4}+\rho^{4}\right) \\
P_{4}(s, \rho) & =\frac{1}{4} & P_{10}(s, \rho) & =\frac{93}{256}+\frac{73}{768} s^{2}-\frac{47}{256} \rho^{2}-\frac{17}{384} s^{2} \rho^{2}+\frac{11}{768} s^{4} \\
P_{6}(s, \rho) & =\frac{5}{16}+\frac{1}{16}\left(s^{2}-\rho^{2}\right) & & +\frac{23}{768} \rho^{4}+\frac{1}{256}\left(s^{2} \rho^{4}-s^{4} \rho^{2}\right)+\frac{1}{768}\left(s^{6}-\rho^{6}\right) .
\end{aligned}
$$

Extending the results to the $3 \mathrm{D}$ case we may define the radial variables as

$$
s=\left\{\begin{array}{ll}
\left|s_{y}\right| & \text { for } 2 \mathrm{D} \\
\sqrt{s_{y}^{2}+s_{z}^{2}} & \text { for } 3 \mathrm{D}
\end{array} \quad \text { and } \quad \rho= \begin{cases}\left|\rho_{x}\right| & \text { for } 2 \mathrm{D} \\
\left|\rho_{x}\right| & \text { for } 3 \mathrm{D}\end{cases}\right.
$$

For the case of $s=0$ we may define the Green's function arbitrarily as it represents the average value of the solution which for a potential is arbitrarily defined. In this work we have used $\widehat{G}_{m}(\rho, 0)=0$. We furthermore notice that for $\sigma \rightarrow 0$ the regularized Green's function (Eq. (15)) reduces to the non-regularized Green's function obtained by Chatelain and Koumoutsakos [22].

The regularized Green's function for the Poisson equation in 3D for the case with one periodic $(z)$ and two unbounded directions ( $x$ and $y$ ) may be derived following the approach above. The Fourier transform of the Green's function in the periodic $z$-direction is the $2 \mathrm{D}$ radial inverse Fourier transform

$$
\widehat{G}_{m}\left(\rho, s_{z}\right)=\frac{1}{2 \pi} \int_{0}^{\infty} \frac{\widehat{\zeta}_{m}\left(\sqrt{s^{2}+s_{z}^{2}}\right)}{s^{2}+s_{z}^{2}} J_{0}(s \rho) s d s,
$$

where $s^{2}=s_{x}^{2}+s_{y}^{2}$ and $\rho^{2}=\rho_{x}^{2}+\rho_{y}^{2}$.

It is however not trivial to find a closed form expression of the integral Eq. (18), but we notice that to compute discrete solutions to Eq. (5) using the FFT-based Poisson solver a regular spectral representation is sufficient. The integrand of Eq. (18) is regular for $s_{z}^{2} \neq 0$ and may be used as it is stated. For $s_{z}^{2}=0$ it may be expressed through the completely unbounded regularised Green's function in 2D proposed by Hejlesen et al. [8]

$$
\begin{aligned}
\widehat{G}_{m}\left(s, s_{z}\right)= \begin{cases}\widehat{G}_{m}^{2 \mathrm{D}}(s) \frac{D_{m}\left(\sqrt{s^{2}+s_{z}^{2}}\right)}{D_{m}\left(\sqrt{s^{2}}\right)} \exp \left(-\frac{s_{z}^{2}}{2}\right) & \text { for } s_{z}=0 \\
\frac{\widehat{\zeta}_{m}\left(\sqrt{s^{2}+s_{z}^{2}}\right)}{s^{2}+s_{z}^{2}} & \text { for } \quad s_{z} \neq 0\end{cases} \\
G_{m}^{2 \mathrm{D}}(\rho)=-\frac{1}{2 \pi}\left(\ln (\sigma \rho)-Q_{m}(\rho) \exp \left(-\frac{\rho^{2}}{2}\right)+\frac{1}{2} E_{1}\left(\frac{\rho^{2}}{2}\right)\right),
\end{aligned}
$$


where $\widehat{G}_{m}^{2 \mathrm{D}}(s)$ is the 2D radial Fourier transform of $G_{m}^{2 \mathrm{D}}(\rho)$ and $Q_{m}(\rho)$ is a polynomial given in [8]

$$
\begin{aligned}
Q_{2}(\rho)=1 & Q_{6}(\rho)=1-\rho^{2}+\frac{1}{8} \rho^{4} \\
Q_{4}(\rho)=1-\frac{1}{2} \rho^{2} \quad & Q_{8}(\rho)=1-\frac{3}{2} \rho^{2}+\frac{3}{8} \rho^{4}-\frac{1}{48} \rho^{6} \\
Q_{10}(\rho) & =1-2 \rho^{2}+\frac{3}{4} \rho^{4}-\frac{1}{12} \rho^{6}+\frac{1}{384} \rho^{8}
\end{aligned}
$$

The Fourier coefficients of Eq. (19) may in principle be computed faster than those of [22] as the computation involves the FFT of a single plane only, but in a parallel implementation, it may be difficult to exploit this. The method however may be used with the spectral accurate 2D unbounded kernels by [10, 26] or [27]. The kernel by [27] is conveniently given in Fourier-space but it impose a zero-padding in a precomputation step by a length in each direction greater than or equal to the maximum distance between any two particles in the computational domain. Hence may not be feasible for aspect ratios larger than one.

\section{Results}

\subsection{Verification of accuracy}

To investigate the accurate and consistency of the FFT-based Poisson solver with mixed boundary conditions, we must consider a test function that is bounded within the computational domain in the unbounded directions, and fully continuously differentiable in the periodic directions. The test function must furthermore have at least two continuous derivatives which is a direct consequence of the mapping of the Laplace operator $\nabla^{2}: C^{m} \rightarrow C^{m-2}$ after which $m \geq 2$ is needed to give a continuous solution for the Poisson equation. However, in order to make the quadrature error insignificant and thus allowing an arbitrary high convergence rate the test function must be infinitely differentiable.

To meet these requirements we use a sine function for the periodic directions and a normalized bump function distribution in the unbounded directions, which both are of class $C^{\infty}$ i.e. it has an infinite number of continuous derivatives. The bump function is defined as

$$
f(q)= \begin{cases}\exp \left[c\left(1-\frac{1}{1-q^{2}}\right)\right] & \text { for } \quad|q|<1 \\ 0 & \text { for }|q| \geq 1\end{cases}
$$

where $c$ is an arbitrary positive constant. For the presented cases $c=10$ is used.

For the $2 \mathrm{D}$ case with one unbounded $(x)$ and one periodic $(y)$ directions, we solve the scalar equation of Eq. (5) using the test function solution

$$
\phi(x, y)=f\left(\frac{x}{R}\right) \sin (2 \pi y)
$$


Similarly, for the 3D case with one unbounded $(x)$ and two periodic ( $y$ and $z$ ) directions, we use the test function solution

$$
\phi(x, y, z)=f\left(\frac{x}{R}\right) \sin (2 \pi y) \sin (2 \pi z)
$$

For the 3D case with two unbounded $(x$ and $y)$ and one periodic $(z)$ directions, we use a test function solution which is non-zero when averaged in the periodic direction to verify a consistent treatment of singularity at $s_{z}=0$

$$
\phi(x, y, z)=f\left(\frac{\sqrt{x^{2}+y^{2}}}{R}\right)[1+\sin (2 \pi z)] .
$$

We solve the scalar equation and define the error of the calculated vector field $\vartheta_{i}$ compared to the analytic solution which is sampled in the same points $\vartheta\left(\boldsymbol{x}_{i}\right)$ as

$$
\text { error }=\left(\sum_{i=1}^{N}\left|\vartheta\left(\boldsymbol{x}_{i}\right)-\vartheta_{i}\right|^{2} / \sum_{i=1}^{N}\left|\vartheta\left(\boldsymbol{x}_{i}\right)\right|^{2}\right)^{1 / 2} \text {. }
$$

The obtained error for the regularized Poisson solver with mixed boundary conditions is shown in Fig. 1 compared to the non-regularized method of Chatelain and Koumoutsakos [22] in 3D for a single periodic direction.

Also we compare to results obtained by point to point evaluations of the analytic Green's function for the $2 \mathrm{D}$ problem by Lamb $[28,29]$ written in complex notation as:

$$
G(\boldsymbol{x})=-\frac{1}{2 \pi} \log \left(\sin \frac{\pi(z)}{L}\right), \quad z=x+\iota y .
$$

\footnotetext{
It is seen that the method obtains the convergence rate corresponding to the respective design parameters of the Green's functions. The regularized method is shown to quickly produce an error which is significantly lower than that of $[22]$ and $[28,29]$ in the 2D case for $m>2$. Similarly the regularized method shows lower error in 3D for all tested resolutions for $m>2$. Using $m=10$ gives an improvement in accuracy by several orders from the coarsest resolution.

\subsection{Instability of counter rotating vortex pairs}

We illustrate an application of the FFT-based Poisson solver for a problem with mixed boundary conditions (two unbounded and one periodic direction) by simulating the evolution of two counter rotating vortex pairs from an initial state following [30, 31, 22].

The vorticity transport equation, which governs incompressible fluid motion is given by

$$
\frac{D \omega}{D t}=(\boldsymbol{\omega} \cdot \nabla) \boldsymbol{v}+\nu \nabla^{2} \boldsymbol{\omega}
$$

where $\boldsymbol{\omega}=\boldsymbol{\nabla} \times \boldsymbol{v}$. Eq. (28) is discretized using a vortex particle mesh method; the vorticity $(\boldsymbol{\omega})$ is sampled onto a set of discrete particles carrying vorticity $\left(\boldsymbol{\omega}_{p}\right)$. The particle vorticity is interpolated to a uniform 
a)

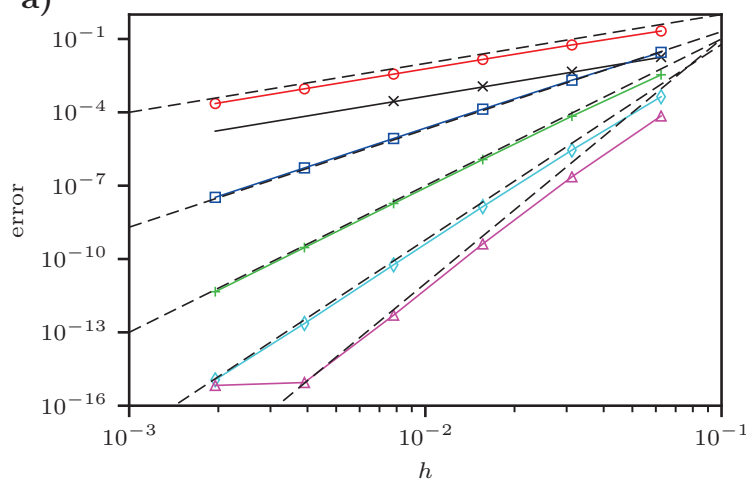

b)

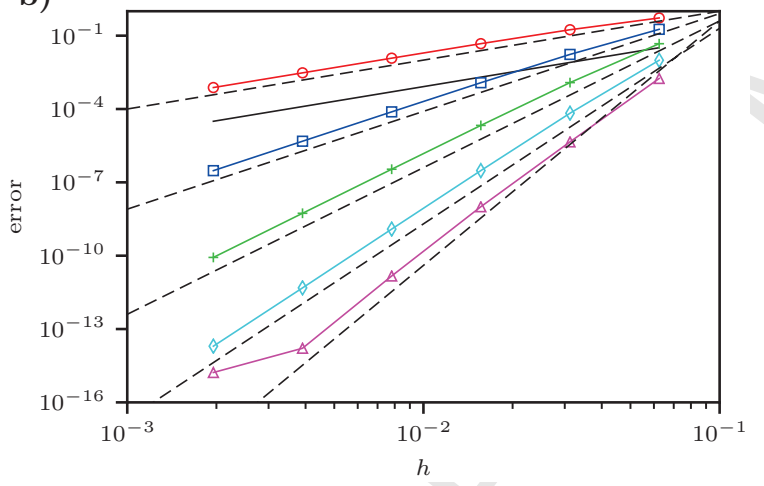

c)

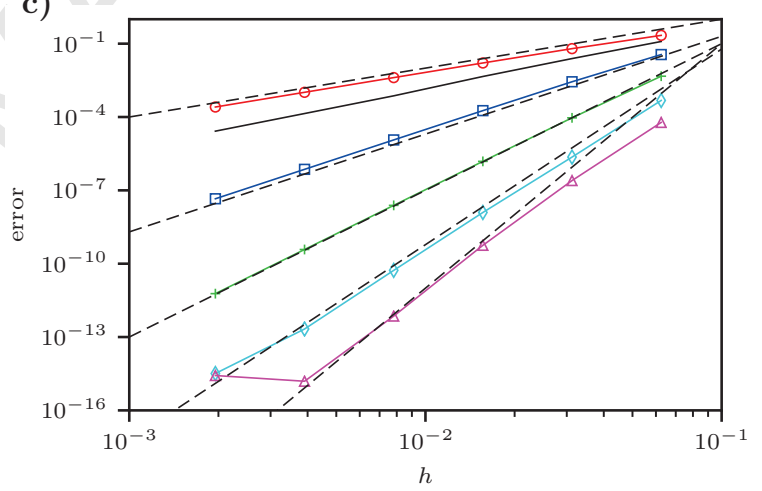

Figure 1: The error of the calculated solutions obtained by the $m$-th order regularized Green's functions: (a) 2D problem with one unbounded and one periodic direction; (b) 3D problem with one unbounded and two periodic directions. (c) 3D problem with two unbounded and one periodic direction. Lines indicate: convergence order (-- - ) from top $\mathcal{O}\left(h^{2}\right), \mathcal{O}\left(h^{4}\right)$, $\mathcal{O}\left(h^{6}\right), \mathcal{O}\left(h^{8}\right), \mathcal{O}\left(h^{10}\right)$; point to point evaluation with the Green's function by Lamb [28, 29]: (× ); the non-regularized Green's function $[22]:(-) ; m=2:(\neg) ; m=4:(\neg) ; m=6:(\neg) ; m=8:(\neg) ; m=10:(\neg)$. 
grid using a third order accurate interpolation kernel [32]. We use the FFT-based Poisson solver to compute the velocity $(\boldsymbol{v})$ by applying the Helmholtz decomposition to the velocity as Eq. (4) and solve for the vector potential in Eq. (5) followed by a spectral approximation to the curl. For the Poisson solver we use a tenth order accurate Green's function with $\alpha=2$. The discrete approximation is evolved in the Lagrangian frame of reference by applying a second order explicit Runge-Kutta time stepping scheme to

$$
\begin{aligned}
\frac{\mathrm{d} \boldsymbol{x}_{p}}{\mathrm{~d} t} & =\boldsymbol{v}\left(\boldsymbol{x}_{p}, t\right) \\
\frac{\mathrm{d} \boldsymbol{\omega}_{p}}{\mathrm{~d} t} & =\left[(\boldsymbol{\omega} \cdot \boldsymbol{\nabla}) \boldsymbol{v}+\nu \boldsymbol{\nabla}^{2} \boldsymbol{\omega}\right]_{p} .
\end{aligned}
$$

Further we enforce a divergence free vorticity field when necessary through a projection step based on the Helmholtz decomposition (Eq. (4)). This is done whenever a norm of the vorticity divergence exceeds a certain threshold, which happens every few steps in the current example (for the details see [33]).

The initial vorticity field consists of two counter rotating vortex-pairs given as

$$
\begin{aligned}
\boldsymbol{\omega} & =\sum_{i}^{2} \frac{\Gamma_{i}}{\pi}\left(\frac{\phi}{p_{i}^{2}+\phi^{2}}\right)^{2} \boldsymbol{e}_{z}-\sum_{i}^{2} \frac{\Gamma_{i}}{\pi}\left(\frac{\phi}{q_{i}^{2}+\phi^{2}}\right)^{2} \boldsymbol{e}_{z}, \\
p_{i}^{2} & =\left(x+b_{i}\right)^{2}+(y-\epsilon)^{2}, \quad q_{i}^{2}=\left(x-b_{i}\right)^{2}+(y-\epsilon)^{2},
\end{aligned}
$$

where $z$ is the periodic direction and $p_{i}, q_{i}$ are the perturbed radii of the respective vortices in the unbounded plane. Following $[30,31,22]$ we use $\Gamma_{2} / \Gamma_{1}=-0.3, b_{2} / b_{1}=0.3, \phi_{1} / b_{1}=0.075$ and $\phi_{2} / b_{1}=0.05$ and define the timescale and the Reynolds number based on the initial circulation $\left(\Gamma_{0}=\Gamma_{1}+\Gamma_{2}\right)$ and centroid $\left(b_{0}=\left[\Gamma_{1} b_{1}+\Gamma_{2} b_{2}\right] / \Gamma_{0}\right)$

$$
T=2 \pi b_{0}^{2} / \Gamma_{0}, \quad \operatorname{Re}=\Gamma_{0} / \nu=3500 .
$$

The system is primarily unstable to the mode of wave length $\lambda_{z} / b_{0}=0.76[30,31]$. To onset the instability the perturbation is set as $\epsilon(z)=10^{-5} b_{0} \sin \left(2 \pi z / \lambda_{z}\right)$. The computational domain spans a single wave length of this mode in the periodic direction $\left(L_{z}=\lambda_{z}\right)$ and the span in the unbounded directions is adapted to encapsulate vortex particles for which $\left|\boldsymbol{\omega}_{p}\right|>10^{-4}|\boldsymbol{\omega}|_{\max }$.

We measure the time variation of the enstrophy $(\Omega)$ and the distribution of kinetic energy among wave numbers $\left(E\left(k_{z}\right)\right)$

$$
\begin{aligned}
\Omega & =\int \boldsymbol{\omega}(x, y, z) \cdot \boldsymbol{\omega}(x, y, z) d V \\
E\left(k_{z}\right) & =\iint \frac{1}{2}\left(\widehat{\boldsymbol{\Psi}}^{*}\left(x, y, k_{z}\right) \cdot \widehat{\boldsymbol{\omega}}\left(x, y, k_{z}\right)+\widehat{\boldsymbol{\omega}}^{*}\left(x, y, k_{z}\right) \cdot \widehat{\boldsymbol{\Psi}}\left(x, y, k_{z}\right)\right) d x d y,
\end{aligned}
$$

where $(\cdot)^{*}$ denotes the complex conjugate.

The evolution of enstrophy is shown in Fig. 2(a). It may be seen that that the obtained results converge (with mesh refinement) towards the same solution as obtained in [22] (for $h / L_{z}=256$ ). Furthermore the 
a)

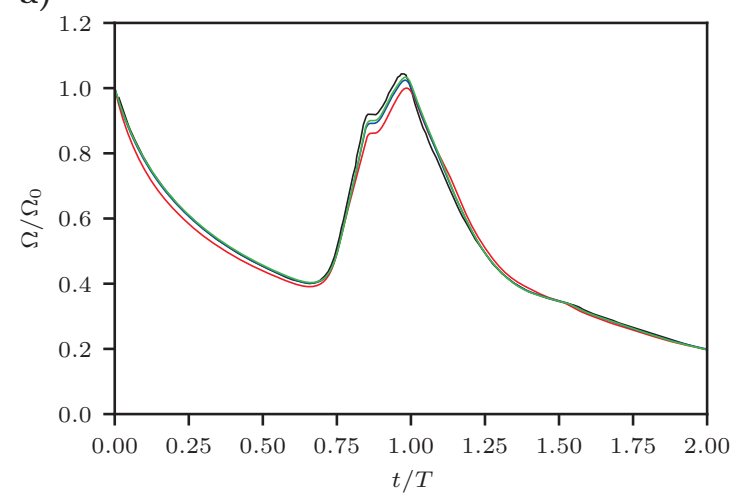

b)

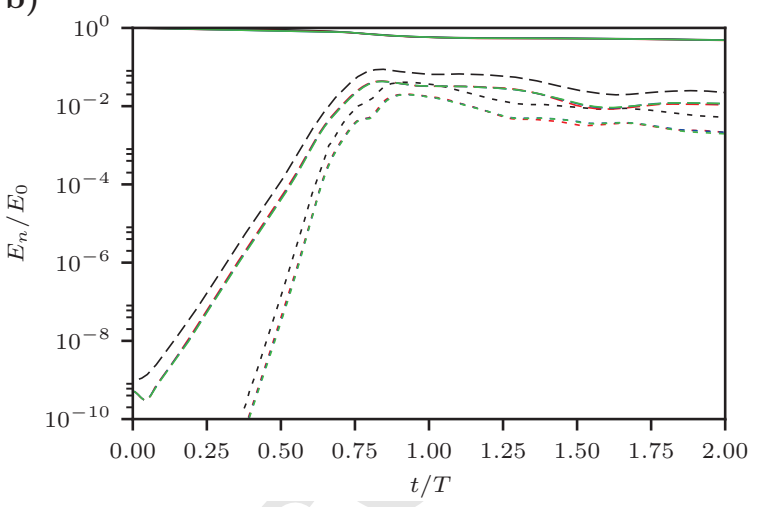

Figure 2: (a) Evolution of enstrophy. (b) Evolution of kinetic energy associated with first three modes: $0^{\text {th }}:\left(\right.$ solid); $1^{\text {st }}:($ dashed); $2^{\text {nd }}$ (dotted). Line colors indicate results from: [22]: $(-)$; simulation using $h / L_{z}=64$ : $(-)$; simulation using $h / L_{z}=$ 128: ( - ); simulation using $h / L_{z}=256:(-)$.

time history of kinetic energy associated with first three modes (Fig. 2(b)) displays very similar growth rates as in [22] though the current results are slightly delayed possible due different perturbation applied or other variations in the algorithms (e.g., time stepping and projection-scheme). Iso contours of the vorticity magnitude is shown for different time instances in Fig. 3. These shows a transition from a 2D state into a 3D state primarily through the perturbed wave length. The 3D dynamic involves a stretching of one pair member around the other and creation of smaller scales in the solution through non-linear interaction.

\section{Conclusion}

Regularized Green's functions for domains with a mixture of unbounded and periodic directions has been derived. The regularization of the Green's function removes its singularity by introducing a regularization radius which is related to the discretization length and hence imposes a minimum resolved scale. The regularized unbounded-periodic Green's functions has been implemented in a high order FFT-based Poisson solver for domains with a mixture of unbounded and periodic directions. The Poisson solver has been shown to results in a convergence rate corresponding to the regularization order of the Green's function and its applicability has been demonstrated in a vortex particle mesh method. The high order is achieved without any additional computational cost from the conventional FFT-based Poisson solver and is able to calculate the derivative of the solution to the same high order by direct spectral differentiating.

\section{Acknowledgement}

We would like to acknowledge the helpful discussions with Johannes Tophøj Rasmussen, Philippe Chatelain and Grégoire Winckelmans. Further, we thank the reviewers for their insightful and helpful comments. 


\section{ACCEPTED MANUSCRIPT}

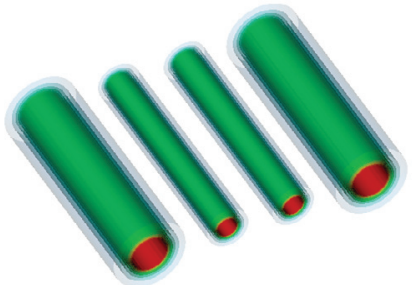

$t / T=0.0$
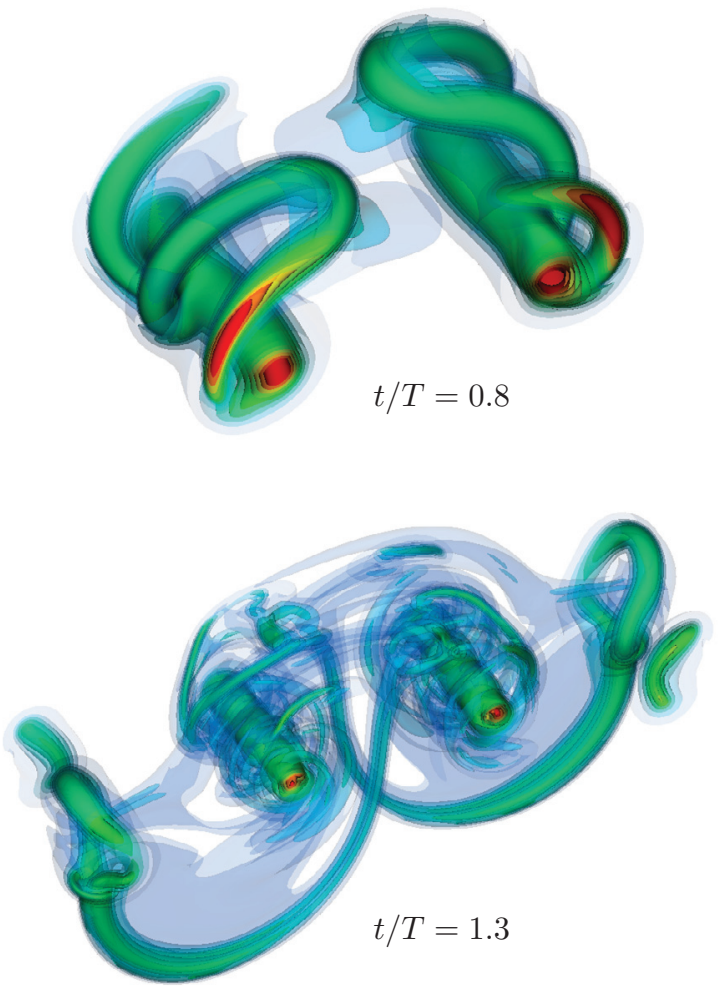

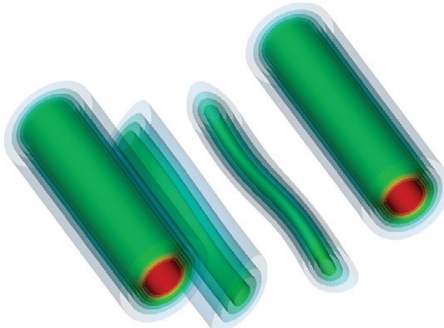

$t / T=0.6$
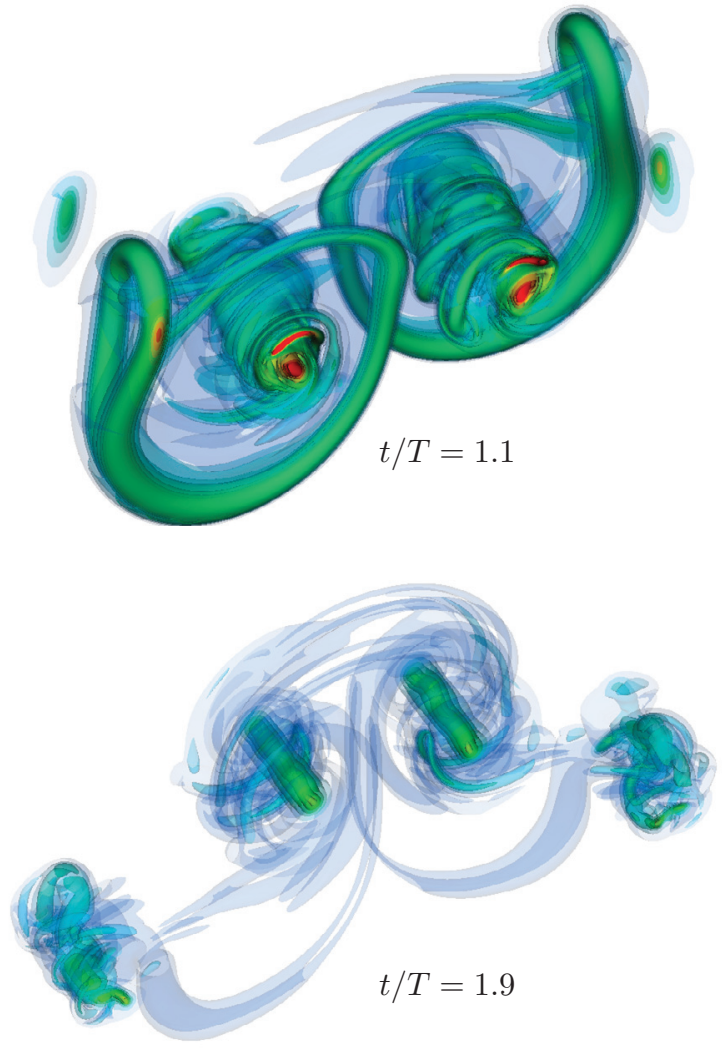

Figure 3: Isocontours of the vorticity magnitude from blue to red $\left|\boldsymbol{\omega} b_{1}^{2} / \Gamma_{0}\right|=[1,4,8,12,16,20,24,28,32]$ at various times. 
This research was funded by the Danish Council for Independent Research grant no. 4184-00349B.

[1] P. P. Ewald, Die Berechnung Optischer und Elektrostatische Gitterpotentiale, Annalen der Physik 64 (1921) $253-287$.

[2] R. W. Hockney, J. W. Eastwood, Computer Simulation Using Particles, 2nd Edition, Institute of Physics Publishing, Bristol, PA, USA, 1988.

[3] T. Darden, D. York, L. Pedersen, Particle mesh Ewald: An $N \cdot \log N$ method for Ewald sums in large systems, J. Chem. Phys. 98 (12) (1993) 10089-10092.

[4] U. Essmann, L. Perera, M. L. Berkowitz, T. Darden, H. Lee, L. G. Pedersen, A smooth particle mesh Ewald method, J. Chem. Phys. 103 (19) (1995) 8577-8593.

[5] A.-K. Tornberg, The Ewald sums for singly, doubly and triply periodic electrostatic systems, Advances in Computational Mathematics 42 (1) (2016) 227-248.

[6] D. Lindbo, A.-K. Tornberg, Spectral accuracy in fast Ewald-based methods for particle simulations, J. Comput. Phys. 230 (2011) 8744-8761.

[7] F. Nestler, M. Pippig, D. Potts, Fast Ewald summation based on NFFT with mixed periodicity, J. Comput. Phys. 285 (2015) 280-315.

[8] M. M. Hejlesen, J. T. Rasmussen, P. Chatelain, J. H. Walther, A high order solver for the unbounded Poisson equation, J. Comput. Phys. 252 (2013) 458-467.

[9] M. M. Hejlesen, J. T. Rasmussen, P. Chatelain, J. H. Walther, High order Poisson solver for unbounded flows, Procedia IUTAM 18 (2015) 56-65.

[10] M. M. Hejlesen, A high order regularisation method for solving the Poisson equation and selected applications using vortex methods, Ph.D. thesis, Technical University of Denmark (February 2016).

[11] A. Leonard, Vortex methods for flow simulation, J. Comput. Phys. 37 (1980) 289-335.

[12] J. T. Beale, A. Majda, High order accurate vortex methods with explicit velocity kernels, J. Comput. Phys. 58 (1985) $188-208$.

[13] M. Perlman, On the accuracy of vortex methods, J. Comput. Phys. 59 (1985) 200-223.

[14] O. H. Hald, Convergence of vortex methods for Euler's equations, III, SIAM J. Numer. Anal. 24 (3) (1987) 538-582.

[15] G. S. Winckelmans, A. Leonard, Contribution to vortex particle methods for the computation of three-dimensional incompressible unsteady flows, J. Comput. Phys. 109 (1993) 247-273.

[16] L. Greengard, V. Rokhlin, A fast algorithm for particle simulations, J. Comput. Phys. 73 (1987) $325-348$.

[17] J. Carrier, L. Greengard, V. Rokhlin, A fast adaptive multipole algorithm for particle simulations, SIAM J. Sci. Stat. Comput. 9 (4) (1988) 669-686.

[18] A. Gholami, D. Malhotra, H. Sundar, G. Biros, FFT, FMM, or multigrid? a comparative study of state-of-the-art Poisson solvers for uniform and nonuniform grids in the unit cube, SIAM J. Sci. Comput. 38 (3) (2016) C280-C306.

[19] H. Langston, L. Greengard, D. Zorin, A free-space adaptive FMM-based PDE solver in three dimensions, Commun. Appl. Math. Comput. Sci. 6 (1) (2011) 79-122.

[20] D. Malhotra, G. Biros, PVFMM: A parallel kernel independent FMM for particle and volume potentials, Communications in Computational Physics 18 (3) (2015) 808-830.

[21] G.-H. Cottet, P. Koumoutsakos, Vortex Methods - Theory and Practice, Cambridge University Press, New York, 2000.

[22] P. Chatelain, P. Koumoutsakos, A Fourier-based elliptic solver for vortical flows with periodic and unbounded directions, J. Comput. Phys. 229 (2010) 2425-2431.

[23] C. R. Anderson, A recursive expanding domain method for the solution of Laplace's equation in infinity domains, CAM report 14-45, University of California (2014).

[24] R. Anderson, Christopher, High order expanding domain methods for the solution of Poisson's equation in infinite domains, J. Comput. Phys. 314 (2016) 194-205. 
[25] J. T. Rasmussen, Particle methods in bluff body aerodynamics, Ph.D. thesis, Technical University of Denmark (October 2011).

[26] M. M. Hejlesen, G. Winckelmans, J. H. Walther, Non-singular Green's functions for the unbounded Poisson equation in 2d and 3d derived using spectral cut-off regularization, arXiv:1704.00704v1 (2017) 1-5.

[27] F. Vico, L. Greengard, M. Ferrando, Fast convolution with free-space Green's functions, J. Comput. Phys. 323 (2016) $191-203$.

[28] S. H. Lamb, Hydrodynamics, 6th Edition, Dover Publication Inc., 1932.

[29] L. Rosenhead, The formation of vortices from a surface of discontinuity, Proc. R. Soc. Lond. A 134 (1931) $170-192$.

[30] G. Winckelmans, R. Cocle, L. Dufresne, R. Capart, Vortex methods and their application to trailing wake vortex simulations, C. R. Physique 6 (2005) 467-486.

[31] P. Chatelain, A. Curioni, M. Bergdorf, D. Rossinelli, W. Andreoni, P. Koumoutsakos, Billion vortex particle direct numerical simulations of aircraft wakes, Comp. Meth. Appl. Mech. \& Engng. 197 (2008) 1296-1304.

[32] J. J. Monaghan, Extrapolating B splines for interpolation, J. Comput. Phys. 60 (2) (1985) 253-262.

[33] H. J. Spietz, M. M. Hejlesen, J. H. Walther, Iterative Brinkman penalization for simulation of impulsively started flow past a sphere and a circular disk, J. Comput. Phys. 336 (2017) 261-274. 